\title{
How to Perform and Interpret Functional Magnetic Resonance Imaging Studies in Functional Gastrointestinal Disorders
}

\author{
In-Seon Lee, ${ }^{1,2}$ Hubert Preissl, ${ }^{3,4}$ and Paul Enck ${ }^{1 *}$ \\ ${ }^{I}$ Psychosomatic Medicine and Psychotherapy Department, University of Tübingen, Tübingen, Germany; ${ }^{2}$ Graduate Training Centre of \\ Neuroscience, International Max Planck Research School, University of Tübingen, Tübingen, Germany; ${ }^{3}$ Institute for Diabetes Research and \\ Metabolic Diseases of the Helmholtz Center Munich at the University of Tübingen, German Center for Diabetes Research (DZD e.V.), Tübingen, \\ Germany; and ${ }^{4}$ Institute of Pharmaceutical Sciences, Department of Pharmacy and Biochemistry, University of Tübingen, Tübingen, Germany
}

Functional neuroimaging studies have revealed the importance of the role of cognitive and psychological factors and the dysregulation of the brain-gut axis in functional gastrointestinal disorder patients. Although only a small number of neuroimaging studies have been conducted in functional gastrointestinal disorder patients, and despite the fact that the neuroimaging technique requires a high level of knowledge, the technique still has a great deal of potential. The application of functional magnetic resonance imaging (fMRI) technique in functional gastrointestinal disorders should provide novel methods of diagnosing and treating patients. In this review, basic knowledge and technical/practical issues of fMRI will be introduced to clinicians.

(J Neurogastroenterol Motil 2017;23:197-207)

Key Words

Brain; Functional magnetic resonance imaging; Functional neuroimaging; Gastrointestinal diseases

\section{Introduction}

Functional gastrointestinal disorders (FGIDs) are associated with functional and histological changes of gastrointestinal compartments such as gastric motility, visceral sensitivity, and inflammation. Our understanding of the underlying pathophysiological mechanisms is, however, limited. The advent and development of functional neuroimaging techniques in humans has facilitated the investigation of bottom-up processes_-brain activations generated by signals from the periphery—and top-down processes—-the ef- fect of cognitive and psychological factors - in healthy volunteers. Functional neuroimaging is now recognized as an objective and accurate tool in the exploration of the central mechanism of functional disorders. Over the past few years, evidence from functional neuroimaging studies has endorsed the hypothesis that the dysregulation of the brain-gut axis (neuronal and hormonal interactions between the brain and the gut) is a key factor in FGIDs. According to previous reviews, ${ }^{1,2}$ the functional alterations in sensory, emotional, painrelated, and homeostatic brain areas (changes of the brain function in frontal cortex, somatosensory cortex, insula, anterior cingulate cortex, thalamus, hippocampus, and amygdala) are the important

Received: November 8, 2016 Revised: None Accepted: December 19, 2016

(c) This is an Open Access article distributed under the terms of the Creative Commons Attribution Non-Commercial License (http://creativecommons. org/licenses/by-nc/4.0) which permits unrestricted non-commercial use, distribution, and reproduction in any medium, provided the original work is properly cited.

${ }^{*}$ Correspondence: Paul Enck, PhD

University Hospital, Department of Internal Medicine VI, Osianderstr. 5, 72076 Tübingen, Germany Tel: +49-07071-29-89118, Fax: +49-07071-29-4382, E-mail: paul.enck@uni-tuebingen.de 
pathogenic factors in FGIDs. Most present-day studies involve patients with irritable bowel syndrome (IBS) and functional dyspepsia (FD) and although several other functional neuroimaging methods are available, functional magnetic resonance imaging (fMRI) has proved to be the most frequently applied technique. Functional MRI is completely non-invasive, sensitive to task-related or nontask-related (resting state) brain activation, with high spatial (a few millimeters) and acceptable temporal (a few seconds) resolution, and facilitates deep brain structure and brain stem-imaging. Moreover, due to the availability of standard analysis tool boxes and tremendous advances in analysis methods, from univariate to multivariate analysis, fMRI has become increasingly popular in cognitive and clinical neuroscience studies.

In this review, we present the technical and practical issues of fMRI and show its application in FGIDs-related studies - with emphasis on IBS and FD patients - to improve clinicians' understanding of the merits of $\mathrm{fMRI}$ studies as well as of their possible limitations. Subsequently, we also propose future approaches in this field to further knowledge of FGIDs.

\section{Brief Overview of the Functional Magnetic Resonance Imaging Technique}

MRI has already been used to investigate tissue properties. In the 1990s, MRI was also deployed to measure the blood oxygen level dependent (BOLD) contrast in the investigation of functional activations in the brain. ${ }^{3}$ Activation of neurons in the brain leads to the consumption of oxygen as well as to an increased flow of blood in the surrounding area (hemodynamic response). These changes result in magnetic field distortions in the brain tissue. To record these changes, the different relaxation times of the protons are measured by a constant magnetic field (nowadays, most fMRI systems use 1.5-7.0 Tesla, the strength of the constant field is a major determinant of the signal strength) and a superimposed gradient magnetic field. A BOLD fMRI signal (increased signal intensity of $\mathrm{T} 2{ }^{*}$-weighted images) is determined by a combination of blood flow, volume, and relative oxygenated hemoglobin level. The temporal signal recorded by BOLD fMRI (Fig. 1B) lies in the range of seconds and does not correspond directly to neuronal activity, but provides a hemodynamic proxy. For the analysis and interpretation of BOLD fMRI, the hemodynamic response function (HRF; Fig.

A Hemodynamic response function (HRF)

\section{B BOLD signal from a voxel}
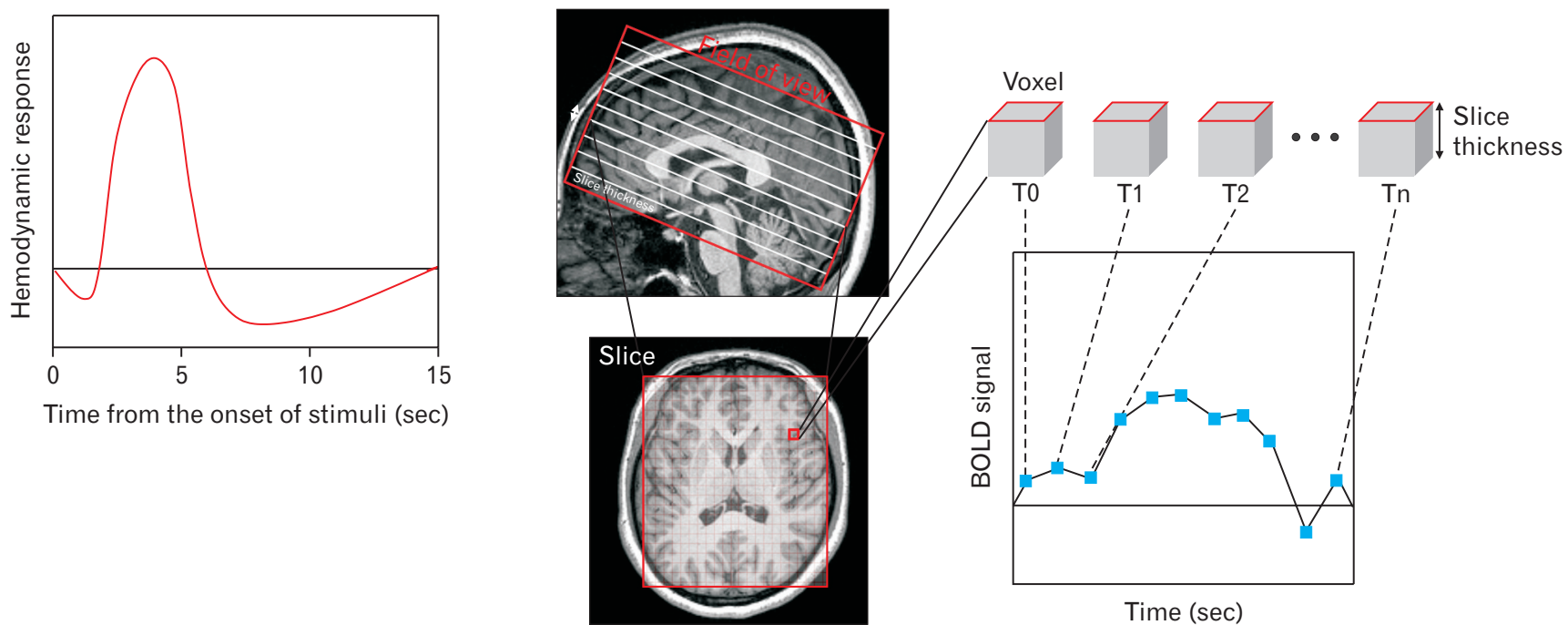

Figure 1. Example of hemodynamic response (A) and time series blood oxygen level dependent (BOLD) signal from a voxel (B). (A) Neurons respond rapidly to internal or external changes and allow the alterations of blood flow and oxygenation in the close area (hemodynamic response) that drives the peak of BOLD signal few seconds after the onset of internal or external changes. BOLD signal slowly returns to baseline level following an undershoot. (B) Within the field of view, each slice consists of a certain number of voxels determined by the size of the measurement matrix. The BOLD signal of each voxel is recorded at consecutive time points and this time trace is further analysed to interfere with functional brain activation. 
1A) that describes the temporal derivative of the BOLD signal related to the neuronal activity must be determined. Most studies now use a homogenous HRF for the whole brain; a fixed model of temporal changes of BOLD signal due to the neuronal activity responding to external stimuli or changes of internal states, which peaks roughly 4-5 seconds after the neuronal event. HRF generates the anticipated BOLD signal which identifies the activation map of brain function (see below, Analysis of Functional Resonance Imaging Image section), and various methods have been proposed with which more spatially or temporally accurate HRF could be retrieved so as to improve fMRI analysis. ${ }^{4,5}$

To derive changes in neuronal activity, relative changes of signal intensity (contrast) are measured rather than absolute fMRI signal intensity. Furthermore, fMRI can be used to obtain not only the relative BOLD signal but also quantitative perfusion measurements. Arterial spin labeling is used to measure the cerebral blood flow by detecting the signal of magnetically labeled arterial blood. ${ }^{6,7}$ The use of a quantitative measure enables us to more easily draw comparisons between studies. In this review, we will focus on BOLD contrast. Glossary of terms for fMRI is summarized in Supplementary Table.

\section{How Is an Functional Magnetic Resonance Imaging Study Performed?}

\section{Design of an Functional Magnetic Resonance Imaging Study}

Not all fMRI study designs are identical, and the designs are adapted depends on the type of research (basic/translational/clinical research, uncontrolled or controlled clinical trials, case reports, etc) and the purpose of the study. At present, most task-related study designs are either block (Fig. 2A) or event-related designs (Fig. 2B). Traditionally, various cognitive tasks, such as perception, attention, learning, memory, language skill, emotion, and motor related tasks, were applied in fMRI studies to identify the location or network of cognitive functions in the brain. However, interest in non-taskrelated brain activations, known as resting-state fMRI (rs-fMRI) in which participants' brain are imaged during resting without any specific tasks, has increased.

\section{Task functional magnetic resonance imaging and resting-state functional magnetic resonance imaging}

In early fMRI studies, fMRI signal responses to the repeated task (or stimulation) during a relatively short time interval were averaged and compared. For example, several blocks of Task A (or Stimulus A) and resting (no task; Fig. 2A, Example 1) or Task A,

A Block design

Example 1

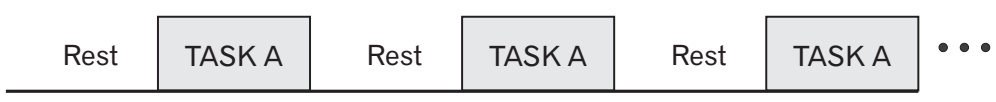

Example 2

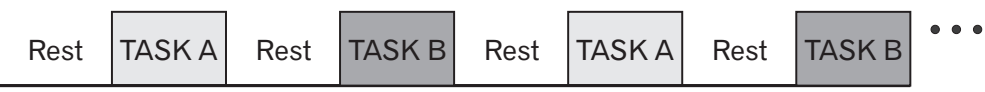

B Event-related design

Example 1

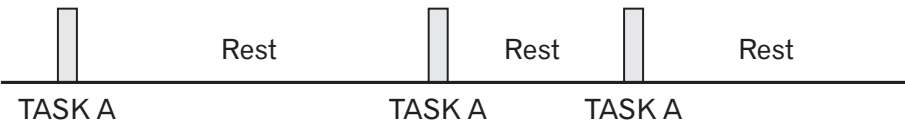

Example 2

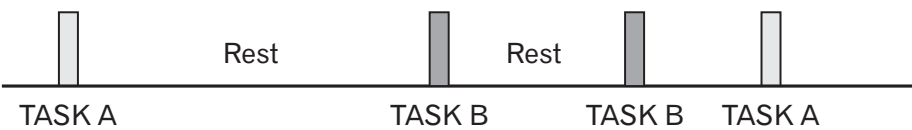

Figure 2. Examples of block design (A) and event-related design (B). (A) Example 1 shows the block design with a single task (Task A) and Example 2 with multiple tasks (Task A, B). (B) Event-related design with a single task (Example 1, Task A) and multiple tasks (Example 2, Task A, B). In both designs, the number of tasks and time durations are laid down in accordance with the type of task, hypothesis, and planned analysis scheme. 
Task B (control condition), and resting (Fig. 2A, Example 2) are presented alternately. In the former case, averaged fMRI signals of blocks of Task A were compared to signals of blocks of resting to show Task A-related increase (Task $\mathrm{A}>$ resting) or decrease (resting $>$ Task A) of BOLD signal in the brain regions. In the latter case, a comparison between the baseline-corrected signals during Tasks A and B revealed that different brain activities were associated with each task. In some cases, two different types of task are delivered simultaneously, eg, pain stimulation during the attention demanding task, ${ }^{8}$ or the basic condition of participants, eg, hunger or satiety, could be modified. ${ }^{9}$ Due to its comparatively high statistical power and large signal changes, block design is an efficient and sensitive method for detecting task-specific brain activations. ${ }^{10,11}$ In a block-design fMRI study, a series of identical tasks (stimuli) are delivered in single block, whereas an event-related design measures the fMRI signal of each single task (stimulation). This approach improves the flexibility of the design by order randomization (which suppresses participants' prediction of the following task) or by posthoc subgroup analysis (eg, correct vs incorrect tasks).

\section{Design of functional magnetic resonance imaging studies in Functional gastrointestinal disorders}

In fMRI studies, visceral distention is the most frequent stimulation performed on patients with FGIDs. The balloon distention method now consists of a bag-type balloon which is placed in an upper or lower gut compartment and distended (supra- or subliminally) by a barostat. ${ }^{12}$ This measures the brain response to visceral stimulation in, for example, patients with IBS. ${ }^{13-44}$ Auditory ${ }^{22,45}$ and somatic pain stimuli ${ }^{19,36}$ were also delivered to patients with IBS in fMRI studies. The results indicate that dysfunction of brain responses in patients is caused not only by visceral sensation but also by non-visceral stimuli, auditory and somatic pain. Cognitive tasks, such as affect matching paradigm, ${ }^{46}$ Wisconsin card sorting test, ${ }^{47}$ emotion recognition paradigm, ${ }^{48}$ and attention network test, ${ }^{49}$ have also been investigated in patients with IBS. Psychological factors such as anxiety and depression were also examined and correlated with brain activation or network parameters in IBS or FD patients. Moreover, $\mathrm{fMRI}$ results were reported as the primary outcome in case report ${ }^{50}$ and clinical trials, ${ }^{37,51,52}$ and brain responses to the treatment itself ${ }^{37,53}$ were examined to ascertain the effect or neuronal mechanisms of pharmacological or non-pharmacological treatments (acupuncture, moxibustion, hypnosis, etc). In such cases, fMRI data were usually obtained before, during, and after the treatment (repeated measurements).

Resting-state $\mathrm{fMRI}$ has already been carried out in a number of studies with IBS $^{54-61}$ and FD patients ${ }^{62-68}$ and its use continues to increase. Functional connectivity, (fractional) amplitude of lowfrequency fluctuations ((f)ALFF), regional homogeneity (ReHo), independent component analysis (ICA), clustering, and graph theory analysis (see below, Advanced analysis) have been used as well as correlation analyses between the effect of adverse history, anxiety and depression, symptom severity, and the brain activity.

\section{Analysis of Functional Magnetic Resonance Image}

The initial goal of fMRI analysis was to identify voxels in the brain that show significant differences between tasks or against rest. In the history of fMRI analysis, great emphasis has always been placed on reducing noise and artifacts and on developing methods to deal with the multiple comparison problem caused by the large number of voxels. The localization of those specific brain regions activated during experimental conditions and its interaction with behavior and cognitive function data (task outcomes, physiological measurements, subjective ratings, questionnaire values, symptom severity, etc) were the primary goals of early fMRI studies (task$\mathrm{fMRI})$. A newly developed approach to fMRI analysis reveals patterns of $\mathrm{AMRI}$ signals such as temporal correlation-based functional connectivity, (f)ALFF, ReHo, ICA, clustering, and graph theory analysis in both task-based and rs-fMRI. For example, if a fluctuation of a time series signal of voxels corresponds to the timing of a certain task in task-based fMRI, then we can detect these voxels with general linear model (GLM). On the basis of the availability of the HRF and the known onset and duration of tasks, an anticipated BOLD signal could be generated (input function $\times \mathrm{HRF}$ $=$ expected BOLD response; Fig. 3A). The expected BOLD signal is utilized to estimate the task-specific activation of voxels. For example, in GLM, the linear relationship between observed (from voxels, dependent variable, blue signal in Fig. 3B) and expected (from HRF, independent variable, red signal in Fig. 3B) BOLD signal is estimated. The voxels whose observed BOLD signal corresponds significantly to the expected BOLD signal, as in Figure $3 \mathrm{~B}$, could be defined as the activated voxels following the task.

The sequence of any fMRI analysis is (1) preprocessing, (2) single subject analysis, (3) group analysis, and (4) additional analysis and visualization. A number of software programs and scripts have been developed for each step of an fMRI analysis. In general, statistical parametric mapping (http://www.fil.ion.ucl.ac.uk/spm/), FMRIB software library (http://fsl.fmrib.ox.ac.uk/fsl/fslwiki/), analysis of functional neuroImages (https://afni.nimh.nih.gov/afni/), 
A Generation of expected BOLD signal using HRF

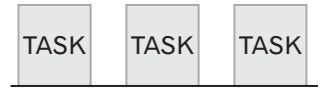

Stimuli input $\bigotimes$

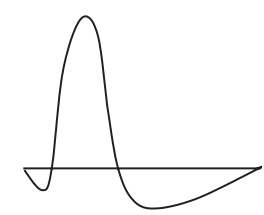

HRF

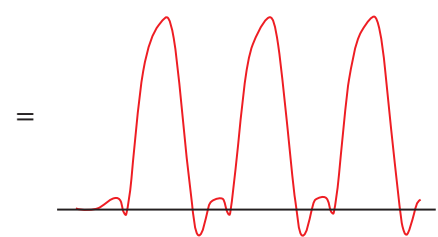

Expected BOLD signal
B Example of observed and expected BOLD signal in block design

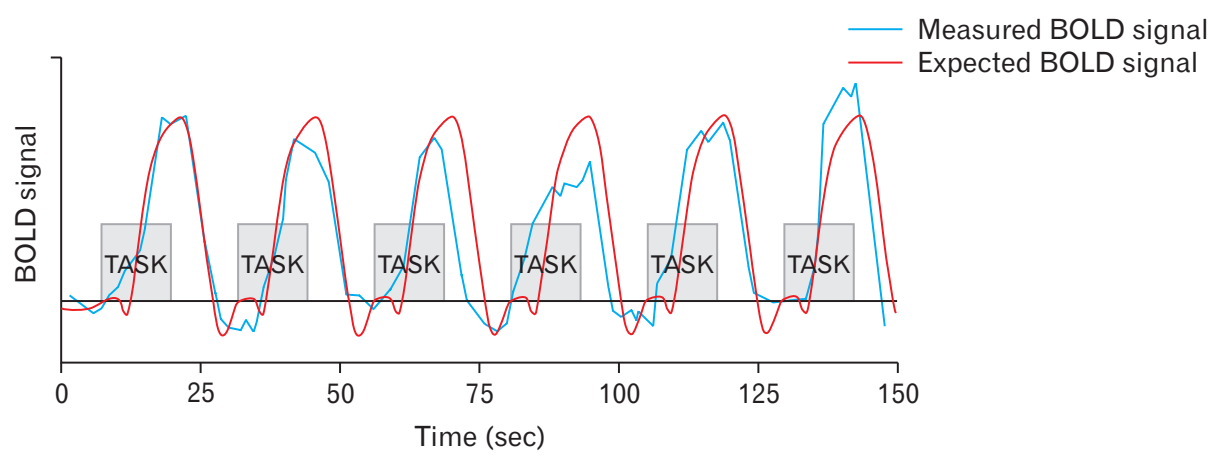

Figure 3. Illustration of expected and measured blood oxygen level dependent (BOLD) signal from single voxel in task functional magnetic resonance imaging. (A) Example of expected BOLD signal using hemodynamic response function (red). (B) Illustration of measured BOLD signal in task-specific activated voxel (blue) and simulated BOLD signal (red) from (A). In the general linear model, the linear relationship between observed (blue) and expected BOLD signal (red) is estimated.
BrainVoyager (http://www.brainvoyager.com/), and additional toolboxes for certain analysis are used. Since the terminology and the steps of analysis differ considerably between the various kinds of software, we will confine ourselves to describing the process of analysis on the basis of the BOLD signal analysis with statistical parametric mapping.

\section{Statistical Power}

As with other types of studies, it is prudent to perform a statistical power analysis before conducting the main fMRI study. To obtain an optimal statistical power (the probability of rejecting the null hypothesis when it is false), it is vital that the effect size and the sample size be taken into consideration. The size of effect is influenced by the sequence parameters, type of task, study design, inter/ intra-variability of the sample data, and the sample size. The latter can easily be controlled by the experimenter. If the anticipated effect size is taken from pilot data or open source data from fMRI databases, a power analysis can be conducted before embarking on the main study to determine the optimal sample size. ${ }^{69,70}$ Desmond and Glover $^{71}$ tested simulated fMRI data to estimate the statistical power. They ascertained that a minimum of 12 subjects is required to ensure $80 \%$ power at $\alpha=0.05$ at the single voxel level and almost twice as many are necessary to achieve the same power level after multiple comparison correction. However, Yarkoni ${ }^{72}$ claimed that the results in $\mathrm{fMRI}$ studies with a small sample size were overestimated and proposed that 50 is a reasonable sample size. At present, sample sizes below 20 are generally considered to be rather small.

\section{Task Functional Magnetic Resonance Imaging}

\section{Preprocessing}

Preprocessing is necessary to modify the recorded fMRI signal into statistical analyzable data by correcting artifacts and noise generated either by the MRI scanner (acquisition timing) or by participants (head motion, inter-participant variability in anatomical features).

(1) Slice timing correction (temporal preprocessing): the brain in the field of view is repeatedly scanned every few seconds and one scan image is composed of several slices (planar image) of the brain. In other words, the slices in one scan image are not collected concurrently (Fig. 1B). To increase the time-sensitive effects, all times series of each slice are adjusted to the acquisition time of one slice (reference slice).

(2) Realignment (spatial preprocessing): participants' head motions, which produce signal noise and voxel mismatch between scans, are corrected. Since larger movements $(>2 \mathrm{~mm},>2$ degree 
rotation) can produce significant non-amendable noise, slices with large head motion are usually discarded. Smaller movements can be corrected or the movement can be taken into consideration during the statistical analysis.

(3) Co-registration (spatial preprocessing): registration of an anatomical image to match the functional image is required for further analysis.

(4) Segmentation (spatial preprocessing): segmentation of an anatomical image to separate brain tissues, cerebral spinal fluid, white matter, and gray matter.

(5) Normalization: individual images are normalized into standard space to correct between subject variability. This step increases sensitivity, and facilitates the generalization of results and comparisons between studies.

(6) Smoothing: a smoothing filter, such as Gaussian kernel, is applied to blur the images and reduce the number of independent observations based on random field theory. This process suppresses noise, increases sensitivity, and makes images more appropriate for single-subject and group analysis.

\section{Single subject and group analysis}

In a single subject analysis, also known as subject level or first level analysis, design and contrast of all experimental conditions are defined. In order to specify the experimental design, information about the onset and duration of each task is required. F-contrasts (effects of interest) or T-contrasts (the contrasts between tasks or task and resting condition) are defined according to the design and purpose of the analysis. Movement parameters and other regressors are also determined in case they are required.

In group analysis, also known as second level analysis, $\mathrm{t}$ tests, ANOVAs and other general linear model analyses with covariates or regressors can be performed. In the event of a specific hypothesis about the correlation between the clinical symptoms, task performance, personality, or duration of the disease and brain activation, multiple regression analysis using covariates could identify those brain regions that positively or negatively correlate with the covariates. Contrasts for group analysis must also be defined to report group level results. In general, the analysis is performed as a whole brain analysis. For region-of-interest (ROI) analysis, the equipped ROIs in the toolbox library (Automated Anatomical Labeling atlas ${ }^{73}$ ) or newly generated ROIs using center coordinates and radius or number of voxels are used. A ROI-based approach should be used only if clear hypotheses are available and the multi-comparison correction should be taken into account if more than one ROI is used. Having set a statistical threshold and multiple comparison correc- tion thresholds to correct false positives (family-wise error rate or false discovery rate is generally used), one can export the results into figures, tables, or time series signal data.

\section{Resting-state Functional Magnetic Resonance Imaging}

Once rs-fMRI data is preprocessed in a similar way to taskfMRI, procedures of single subject and group analysis differ from task-fMRI. In resting state analysis, the spontaneous low frequency fluctuation $(0.01-0.10 \mathrm{~Hz})$ is of major interest. Several approaches, including ALFF and (f)ALFF, were developed specifically for rs-fMRI analysis in an effort to extract an amplitude or ratio of spontaneous low frequency fluctuation from the BOLD signal, indicative of a regional intensity of activation. ${ }^{7475}$ Functional connectivity, ReHo, and ICA are also applicable in rs-fMRI as well as in task-fMRI. Further toolboxes and scripts for rs-fMRI were also developed. ${ }^{76,77}$

\section{Advanced Analysis}

Various advanced analyses have been introduced in fMRI analysis. Here, we briefly introduce the analysis technique which has been used of late in FGIDs studies.

Functional connectivity, one of the most widespread analysis techniques, is defined as 'temporal correlation between the different parts (voxels, clusters, or ROIs) of the brain. ${ }^{38,44,56,57,78}$ It enables us to estimate the connection of brain regions and to compare its patterns between groups. Effective connectivity provides us with additional information as to which brain areas induce a direct causal influence over others. ${ }^{48,51,79}$ Dynamic causal modeling is an example of the effective connectivity analysis method and shows how the effective connectivity (causal influence) between brain regions is modulated by experimental conditions. ${ }^{47,80}$ Graph theory analysis, ie, the analysis of the properties of connections (edges) between functionally connected brain regions (nodes) to account for the complex characteristics of a network, is a further form of connectivity analysis. ${ }^{61,68,81} \mathrm{ReHo}$ is basically a voxel-based connectivity analysis that measures the regional similarity of the signals between the specific voxel and its neighboring voxels. s,67,82 $^{59}$

Of all the multivariate analyses applied in FGIDs studies, ICA pattern classification is the most familiar. ${ }^{29,38,58}$ ICA works on the assumption that an fMRI signal is linearly composed of several (spatially or temporally) independent signals, and that the original fMRI signal is separated into independent groups. ${ }^{83}$ Since ICA is one of the data-driven analysis methods, it can reveal an intrinsic structure of the original signal and can therefore also be utilized to generate hypotheses. 


\section{Interpreting Functional Magnetic Resonance Imaging Results in Functional Gastrointesti- nal Disorders}

In most studies, the list of brain regions (coordinates and statistical information) displaying increased or decreased activity in certain conditions or groups is reported in a voxel-wise or a ROIwise manner. In some instances, a group of the brain areas involved in the same function (eg, pain processing) is identified as a 'network.' For example, albeit opinions are deeply divided on this issue, somatosensory cortex, insula, anterior cingulate cortex, and thalamus are termed a 'pain network. ${ }^{84}$ The most frequently reported brain regions in FGIDs studies are the prefrontal cortex, somatosensory cortex, insula, cingulate cortex, and thalamus. The contributory networks to FGIDs are known as the sensory-motor network, salience network, autonomic network, and cognitive/affective network. ${ }^{1,85}$

Functional MRI data may allow us to elucidate the basic neurophysiological and pathophysiological mechanisms in brains which is associated with clinical information. For example, the activation map following rectal balloon distention can indicate the altered neural processing of visceral pain in the somatosensory cortex, frontal cortex, cingulate cortex, insula, thalamus, and (pre)motor cortex with higher pain sensation (visceral hypersensitivity) in patients than in controls. ${ }^{15,17}$ Anxiety and depression were associated with the brain activation in the cingulate cortex and prefrontal cortex ${ }^{28}$ and history of abuse affected the brain activation in the cingulate cortex. ${ }^{27}$ Several studies have attempted to identify the specific mechanisms of treatment ${ }^{86}$ and neuroimaging biomarkers for further disorders. ${ }^{87}$ The inhibition effect of pain-related brain activation in IBS patients by amitriptyline (tricyclic antidepressants) ${ }^{20}$ identified the central mechanism of antidepressants in the reduction of rectal distention pain. The brain activity during acupuncture suggested the modulation of serotonin pathway at insula and the higher cortical regulation of affection as potential neural mechanisms of acupuncture treatment. ${ }^{34}$ Furthermore, correlation analysis between fMRI data and psychological indices such as anxiety and depression may demonstrate the influence of the psychological state on patients. ${ }^{28,35}$ When interpreting the fMRI results on interventions, the blinding issue, changes of symptoms, co-morbidities, quality of life, non-specific effect, and placebo response should also be taken into consideration carefully.

\section{Limitations and Future Approaches of Functional Magnetic Resonance Imaging Studies in Functional Gastrointestinal Disorders}

Functional MRI measurement is not only expensive and time consuming, but also requires extensive skills and resources. Researchers should be aware of the variety of factors which affect the brain imaging results before performing experiments, and it is only when valid tasks or stimuli, well-structured procedures, controlled populations of participants, and proper analyses come together that reliable data can be gained. The unusual environment of MRI must also be taken into consideration. Patients with a metal implant or with claustrophobia should not participate. No movement, particularly no head movement, is permitted inside the scanner. Recent studies have demonstrated in both IBS and in healthy controls that visceral pain perception is higher within the MRI environment than outside. ${ }^{88}$ Investigators and participants must therefore adapt themselves to the MRI environment.

Until now, all neuroimaging studies in FGID have used a correlation approach. This does not permit us to make any causal inference about the direction of influence (central to peripheral, peripheral to central, or both). At present, inconsistent study designs, analysis methods and statistical principles make it difficult to compare or integrate fMRI data in FGIDs across studies using metaanalysis. However, because FGIDs lack biomarkers such as neurohormones, cytokines, and genes, functional neuroimaging may provide further information to elucidate the symptoms in patients. Furthermore, fMRI studies may help us to better fathom the role of emotional feelings and cognitive functions by combined with other neuroimaging techniques or with autonomic response, genetic and epigenetic approaches, and neurotransmitter research to identify key components of the disease, or to differentiate between subtypes.

In summary, fMRI is a unique research tool that provides information on neuronal mechanisms of symptoms and treatment effects in the patient population, and physiological processing in healthy volunteers. It should, however, be utilized prudently in research, and its pros and cons should be weighed up carefully.

Since neuroimaging has been applied in FGIDs for less than twenty years and analysis methods are developing and improving rapidly, future approaches hold tremendous potential. As yet, only experimental pain stimulation and a few cognitive tasks have been implemented in FGIDs patients. Besides the pain and anxiety/depression scores, FGIDs patients may have many other pathological, behavioral and somatic characteristics; such as impaired affective 
memory, heightened vigilance, abnormal eating behavior, increased stress sensitivity, disordered autonomic regulation, dysbiosis of the gut microbiota, additional bowel symptoms such as nausea, bloating, urgency, and autonomic and somatic co-morbidities. It may be advisable to examine the effects of pharmacological or non-pharmacological therapy, and the influence of such therapies on brain activity may help to establish novel treatment strategies. Albeit still a far cry from clinical application, neuroimaging data will nevertheless one day be used to perform subgroup analyses in patients (eg, hypersensitive vs normosensitive or even hyposensitive patients) or to distinguish patients from healthy controls. ${ }^{89}$ The neuroimaging data with more numerous tasks, behavioral measurement, and therapies could improve our understanding of the pathophysiology of FGIDs and lead to more appropriate treatment options for patients in the future.

\section{Conclusions}

The advent of the fMRI technique has not only provided information on regional brain activities and the interaction of different brain areas, but has also improved our understanding of the neuronal changes and its relationship with symptoms and cognitive/affective changes in many patient groups. Although its usage in basic or clinical neuroscience research in FGIDs patients has been reported in only a limited number of studies, and despite its requiring an intensive level of knowledge in neurology, physiology, pathology, physics, and program coding, it does have considerable potential. An accurate understanding and application of $\mathrm{fMRI}$ technique in FGIDs will hopefully lead to new methods of diagnosing and treating patients.

\section{Supplementary Material}

Note: To access the supplementary table mentioned in this article, visit the online version of Journal of Neurogastroenterology and Motility at http://www.jnmjournal.org/, and at https://doi. org/10.5056/jnm16196.

Acknowledgements: The data of this study were presented at the 6th Asian Postgraduate Course on Neurogastroenterology and Motility (APNM) in Seoul, Korea, 2016.

In-Seon Lee is a PhD training fellow of NeuroGUT.

Financial support: Writing of this review was funded by the People Programme of the European Union's Seventh Framework
Programme under REA grant agreement No. 607652 (NeuroGUT).

\section{Conflicts of interest: None.}

Author contributions: In-Seon Lee, Hubert Preissl, and Paul Enck planned the overall concept and frame work of the manuscript; In-Seon Lee wrote the manuscript; and Hubert Preissl and Paul Enck revised the manuscript.

\section{References}

1. Lee IS, Wang H, Chae Y, Preissl H, Enck P. Functional neuroimaging studies in functional dyspepsia patients: a systematic review. Neurogastroenterol Motil 2016;28:793-805.

2. Mayer EA, Naliboff BD, Craig AD. Neuroimaging of the brain-gut axis: from basic understanding to treatment of functional GI disorders. Gastroenterology 2006;131:1925-1942.

3. Ogawa S, Lee TM, Kay AR, Tank DW. Brain magnetic resonance imaging with contrast dependent on blood oxygenation. Proc Natl Acad Sci USA 1990;87:9868-9872.

4. Maleki-Balajoo S, Hossein-Zadeh GA, Soltanian-Zadeh H, Ekhtiari $H$. Locally estimated hemodynamic response function and activation detection sensitivity in heroin-cue reactivity study. Basic Clin Neurosci 2016;7:299-314

5. Puckett AM, Aquino KM, Robinson PA, Breakspear M, Schira MM. The spatiotemporal hemodynamic response function for depth-dependent functional imaging of human cortex. Neuroimage 2016;139:240-248

6. Grade M, Hernandez Tamames JA, Pizzini FB, Achten E, Golay X, Smits M. A neuroradiologist's guide to arterial spin labeling MRI in clinical practice. Neuroradiology 2015;57:1181-1202.

7. Wu WC, St Lawrence KS, Licht DJ, Wang DJ. Quantification issues in arterial spin labeling perfusion magnetic resonance imaging. Top Magn Reson Imaging 2010;21:65-73.

8. Bantick SJ, Wise RG, Ploghaus A, Clare S, Smith SM, Tracey I. Imaging how attention modulates pain in humans using functional MRI. Brain 2002;125(Pt 2):310-319.

9. Führer D, Zysset S, Stumvoll M. Brain activity in hunger and satiety: an exploratory visually stimulated fMRI study. Obesity (Silver Spring) 2008;16:945-950.

10. Buxton RB, Wong EC, Frank LR. Dynamics of blood flow and oxygenation changes during brain activation: the balloon model. Magn Reson Med 1998;39:855-864

11. Friston KJ, Holmes AP, Price CJ, Büchel C, Worsley KJ. Multisubject fMRI studies and conjunction analyses. Neuroimage 1999;10:385-396.

12. Ritchie J. Pain from distension of the pelvic colon by inflating a balloon in the irritable colon syndrome. Gut 1973;14:125-132.

13. Mertz H, Morgan V, Tanner G, et al. Regional cerebral activation in irritable bowel syndrome and control subjects with painful and nonpainful rectal distention. Gastroenterology 2000;118:842-848.

14. Bernstein CN, Frankenstein UN, Rawsthorne P, Pitz M, Summers R, McIntyre MC. Cortical mapping of visceral pain in patients with GI dis- 
orders using functional magnetic resonance imaging. Am J Gastroenterol 2002;97:319-327.

15. Bonaz B, Baciu M, Papillon E, et al. Central processing of rectal pain in patients with irritable bowel syndrome: an fMRI study. Am J Gastroenterol 2002;97:654-661.

16. Verne GN, Himes NC, Robinson ME, et al. Central representation of visceral and cutaneous hypersensitivity in the irritable bowel syndrome. Pain 2003;103:99-110.

17. Yuan YZ, Tao RJ, Xu B, et al. Functional brain imaging in irritable bowel syndrome with rectal balloon-distention by using fMRI. World J Gastroenterol 2003;9:1356-1360.

18. Sidhu H, Kern M, Shaker R. Absence of increasing cortical fMRI activity volume in response to increasing visceral stimulation in IBS patients. Am J Physiol Gastrointest Liver Physiol 2004;287:G425-G435.

19. Wilder-Smith CH, Schindler D, Lovblad K, Redmond SM, Nirkko A. Brain functional magnetic resonance imaging of rectal pain and activation of endogenous inhibitory mechanisms in irritable bowel syndrome patient subgroups and healthy controls. Gut 2004;53:1595-1601.

20. Morgan V, Pickens D, Gautam S, Kessler R, Mertz H. Amitriptyline reduces rectal pain related activation of the anterior cingulate cortex in patients with irritable bowel syndrome. Gut 2005;54:601-607.

21. Kwan CL, Diamant NE, Pope G, Mikula K, Mikulis DJ, Davis KD. Abnormal forebrain activity in functional bowel disorder patients with chronic pain. Neurology 2005;65:1268-1277.

22. Andresen V, Bach DR, Poellinger A, et al. Brain activation responses to subliminal or supraliminal rectal stimuli and to auditory stimuli in irritable bowel syndrome. Neurogastroenterol Motil 2005;17:827-837.

23. Lawal A, Kern M, Sidhu H, Hofmann C, Shaker R. Novel evidence for hypersensitivity of visceral sensory neural circuitry in irritable bowel syndrome patients. Gastroenterology 2006;130:26-33.

24. Song GH, Venkatraman V, Ho KY, Chee MW, Yeoh KG, Wilder-Smith $\mathrm{CH}$. Cortical effects of anticipation and endogenous modulation of visceral pain assessed by functional brain MRI in irritable bowel syndrome patients and healthy controls. Pain 2006;126:79-90.

25. Price DD, Craggs J, Verne GN, Perlstein WM, Robinson ME. Placebo analgesia is accompanied by large reductions in pain-related brain activity in irritable bowel syndrome patients. Pain 2007;127:63-72.

26. Berman SM, Naliboff BD, Suyenobu B, et al. Reduced brainstem inhibition during anticipated pelvic visceral pain correlates with enhanced brain response to the visceral stimulus in women with irritable bowel syndrome. J Neurosci 2008;28:349-359.

27. Ringel Y, Drossman DA, Leserman JL, et al. Effect of abuse history on pain reports and brain responses to aversive visceral stimulation: an fMRI study. Gastroenterology 2008;134:396-404.

28. Elsenbruch S, Rosenberger C, Enck P, Forsting M, Schedlowski M, Gizewski ER. Affective disturbances modulate the neural processing of visceral pain stimuli in irritable bowel syndrome: an fMRI study. Gut 2010;59:489-495.

29. Hall GB, Kamath MV, Collins S, et al. Heightened central affective response to visceral sensations of pain and discomfort in IBS. Neurogastroenterol Motil 2010;22:276, e80.

30. Elsenbruch S, Rosenberger C, Bingel U, Forsting M, Schedlowski M,
Gizewski ER. Patients with irritable bowel syndrome have altered emotional modulation of neural responses to visceral stimuli. Gastroenterology 2010;139:1310-1319.

31. Larsson MB, Tillisch $\mathrm{K}$, Craig $\mathrm{AD}$, et al. Brain responses to visceral stimuli reflect visceral sensitivity thresholds in patients with irritable bowel syndrome. Gastroenterology 2012;142:463-472, e3.

32. Tillisch K, Labus J, Nam B, et al. Neurokinin-1-receptor antagonism decreases anxiety and emotional arousal circuit response to noxious visceral distension in women with irritable bowel syndrome: a pilot study. Aliment Pharmacol Ther 2012;35:360-367.

33. Lee HF, Hsieh JC, Lu CL, et al. Enhanced affect/cognition-related brain responses during visceral placebo analgesia in irritable bowel syndrome patients. Pain 2012;153:1301-1310.

34. Chu WC, Wu JC, Yew DT, et al. Does acupuncture therapy alter activation of neural pathway for pain perception in irritable bowel syndrome?: a comparative study of true and sham acupuncture using functional magnetic resonance imaging. J Neurogastroenterol Motil 2012;18:305-316.

35. Rosenberger C, Thurling M, Forsting M, Elsenbruch S, Timmann D, Gizewski ER. Contributions of the cerebellum to disturbed central processing of visceral stimuli in irritable bowel syndrome. Cerebellum 2013;12:194-198.

36. Bouhassira D, Moisset X, Jouet P, Duboc H, Coffin B, Sabate JM. Changes in the modulation of spinal pain processing are related to severity in irritable bowel syndrome. Neurogastroenterol Motil 2013;25:623e468.

37. Lowén MB, Mayer EA, Sjöberg M, et al. Effect of hypnotherapy and educational intervention on brain response to visceral stimulus in the irritable bowel syndrome. Aliment Pharmacol Ther 2013;37:1184-1197.

38. Letzen JE, Craggs JG, Perlstein WM, Price DD, Robinson ME. Functional connectivity of the default mode network and its association with pain networks in irritable bowel patients assessed via lidocaine treatment. J Pain 2013;14:1077-1087.

39. Craggs JG, Price DD, Robinson ME. Enhancing the placebo response: functional magnetic resonance imaging evidence of memory and semantic processing in placebo analgesia. J Pain 2014;15:435-446.

40. Schmid J, Langhorst J, Gaß F, et al. Placebo analgesia in patients with functional and organic abdominal pain: a fMRI study in IBS, UC and healthy volunteers. Gut 2015;64:418-427.

41. Zhu Y, Wu Z, Ma X, et al. Brain regions involved in moxibustioninduced analgesia in irritable bowel syndrome with diarrhea: a functional magnetic resonance imaging study. BMC Complement Altern Med 2014;14:500.

42. Icenhour A, Langhorst J, Benson S, et al. Neural circuitry of abdominal pain-related fear learning and reinstatement in irritable bowel syndrome. Neurogastroenterol Motil 2015;27:114-127.

43. Lowén MB, Mayer E, Tillisch K, et al. Deficient habituation to repeated rectal distensions in irritable bowel syndrome patients with visceral hypersensitivity. Neurogastroenterol Motil 2015;27:646-655.

44. Liu X, Silverman A, Kern M, et al. Excessive coupling of the salience network with intrinsic neurocognitive brain networks during rectal distension in adolescents with irritable bowel syndrome: a preliminary report. Neurogastroenterol Motil 2016;28:43-53. 
45. Andresen V, Poellinger A, Tsrouya C, et al. Cerebral processing of auditory stimuli in patients with irritable bowel syndrome. World J Gastroenterol 2006;12:1723-1729.

46. Kilpatrick LA, Labus JS, Coveleskie K, et al. The HTR3A polymorphism c. $-42 \mathrm{C}>\mathrm{T}$ is associated with amygdala responsiveness in patients with irritable bowel syndrome. Gastroenterology 2011;140:1943-1951.

47. Aizawa E, Sato Y, Kochiyama T, et al. Altered cognitive function of prefrontal cortex during error feedback in patients with irritable bowel syndrome, based on fMRI and dynamic causal modeling. Gastroenterology 2012;143:1188-1198.

48. Labus JS, Gupta A, Coveleskie K, et al. Sex differences in emotion-related cognitive processes in irritable bowel syndrome and healthy control subjects. Pain 2013;154:2088-2099.

49. Hubbard CS, Hong J, Jiang Z, et al. Increased attentional network functioning related to symptom severity measures in females with irritable bowel syndrome. Neurogastroenterol Motil 2015;27:1282-1294.

50. Drossman DA, Ringel Y, Vogt BA, et al. Alterations of brain activity associated with resolution of emotional distress and pain in a case of severe irritable bowel syndrome. Gastroenterology 2003;124:754-761.

51. Hubbard CS, Labus JS, Bueller J, et al. Corticotropin-releasing factor receptor 1 antagonist alters regional activation and effective connectivity in an emotional-arousal circuit during expectation of abdominal pain. J Neurosci 2011;31:12491-12500.

52. Zhao JM, Lu JH, Yin XJ, et al. Comparison of electroacupuncture and moxibustion on brain-gut function in patients with diarrhea-predominant irritable bowel syndrome: a randomized controlled trial. Chin J Integr Med 2015;21:855-865.

53. Li Z, Zeng F, Yang Y, et al. Different cerebral responses to puncturing at ST36 among patients with functional dyspepsia and healthy subjects. Forsch Komplementmed 2014;21:99-104.

54. Hong JY, Kilpatrick LA, Labus J, et al. Patients with chronic visceral pain show sex-related alterations in intrinsic oscillations of the resting brain. J Neurosci 2013;33:11994-12002.

55. Gupta A, Kilpatrick L, Labus J, et al. Early adverse life events and resting state neural networks in patients with chronic abdominal pain: evidence for sex differences. Psychosom Med 2014;76:404-412.

56. Hong JY, Kilpatrick LA, Labus JS, et al. Sex and disease-related alterations of anterior insula functional connectivity in chronic abdominal pain. J Neurosci 2014;34:14252-14259.

57. Ma X, Li S, Tian J, et al. Altered brain spontaneous activity and connectivity network in irritable bowel syndrome patients: a resting-state fMRI study. Clin Neurophysiol 2015;126:1190-1197.

58. Gupta A, Rapkin AJ, Gill Z, et al. Disease-related differences in restingstate networks: a comparison between localized provoked vulvodynia, irritable bowel syndrome, and healthy control subjects. Pain 2015;156:809819.

59. Ke J, Qi R, Liu C, et al. Abnormal regional homogeneity in patients with irritable bowel syndrome: a resting-state functional MRI study. Neurogastroenterol Motil 2015;27:1796-1803.

60. Qi R, Liu C, Ke J, et al. Intrinsic brain abnormalities in irritable bowel syndrome and effect of anxiety and depression. Brain Imaging Behav 2016;10:1127-1134
61. Qi R, Ke J, Schoepf UJ, et al. Topological reorganization of the default mode network in irritable bowel syndrome. Mol Neurobiol 2016;53:6585-6593.

62. Zhou G, Liu P, Zeng F, et al. Increased interhemispheric resting-state functional connectivity in functional dyspepsia: a pilot study. NMR Biomed 2013;26:410-415.

63. Zhou G, Liu P, Wang J, et al. Fractional amplitude of low-frequency fluctuation changes in functional dyspepsia: a resting-state fMRI study. Magn Reson Imaging 2013;31:996-1000.

64. Liu P, Zeng F, Zhou G, et al. Alterations of the default mode network in functional dyspepsia patients: a resting-state fmri study. Neurogastroenterol Motil 2013;25:e382-e388.

65. Nan J, Liu J, Li G, et al. Whole-brain functional connectivity identification of functional dyspepsia. PLoS One 2013;8:e65870.

66. Liu P, Qin W, Wang J, et al. Identifying neural patterns of functional dyspepsia using multivariate pattern analysis: a resting-state fMRI study. PLoS One 2013;8:e68205.

67. Nan J, Liu J, Zhang D, et al. Altered intrinsic regional activity and corresponding brain pathways reflect the symptom severity of functional dyspepsia. Neurogastroenterol Motil 2014;26:660-669.

68. Nan J, Zhang L, Zhu F, et al. Topological alterations of the intrinsic brain network in patients with functional dyspepsia. J Neurogastroenterol Motil 2016;22:118-128.

69. Mumford JA. A power calculation guide for fMRI studies. Soc Cogn Affect Neurosci 2012;7:738-742.

70. Joyce KE, Hayasaka S. Development of powermap: a software package for statistical power calculation in neuroimaging studies. Neuroinformatics 2012;10:351-365.

71. Desmond JE, Glover GH. Estimating sample size in functional MRI (fMRI) neuroimaging studies: statistical power analyses. J Neurosci Methods 2002;118:115-128.

72. Yarkoni T. Big correlations in little studies: inflated fMRI correlations reflect low statistical power-commentary on vul et al. (2009). Perspect Psychol Sci 2009;4:294-298

73. Tzourio-Mazoyer N, Landeau B, Papathanassiou D, et al. Automated anatomical labeling of activations in SPM using a macroscopic anatomical parcellation of the MNI MRI single-subject brain. Neuroimage 2002;15:273-289.

74. Zang YF, He Y, Zhu CZ, et al. Altered baseline brain activity in children with ADHD revealed by resting-state functional MRI. Brain Dev 2007;29:83-91.

75. Zou QH, Zhu CZ, Yang Y, et al. An improved approach to detection of amplitude of low-frequency fluctuation (ALFF) for resting-state fMRI: fractional ALFF. J Neurosci Methods 2008;172:137-141.

76. Chao-Gan Y, Yu-Feng Z. DPARSF: a MATLAB toolbox for "pipeline" data analysis of resting-state fMRI. Front Syst Neurosci 2010;4:13

77. Song XW, Dong ZY, Long XY, et al. REST: a toolkit for resting-state functional magnetic resonance imaging data processing. PLoS One 2011;6:e25031.

78. Friston KJ, Frith CD, Liddle PF, Frackowiak RS. Functional connectivity: the principal-component analysis of large (PET) data sets. J Cereb Blood Flow Metab 1993;13:5-14. 
79. Friston KJ. Functional and effective connectivity: a review. Brain Connect 2011;1:13-36.

80. Friston KJ, Harrison L, Penny W. Dynamic causal modelling. Neuroimage 2003;19:1273-1302.

81. Wang J, Zuo X, He Y. Graph-based network analysis of resting-state functional MRI. Front Syst Neurosci 2010;4:16.

82. Zang Y, Jiang T, Lu Y, He Y, Tian L. Regional homogeneity approach to fMRI data analysis. Neuroimage 2004;22:394-400.

83. McKeown MJ, Makeig S, Brown GG, et al. Analysis of fMRI data by blind separation into independent spatial components. Hum Brain Mapp 1998;6:160-188.

84. Apkarian AV, Bushnell MC, Treede RD, Zubieta JK. Human brain mechanisms of pain perception and regulation in health and disease. Eur J Pain 2005;9:463-484.

85. Mayer EA, Labus JS, Tillisch K, Cole SW, Baldi P. Towards a systems view of IBS. Nat Rev Gastroenterol Hepatol 2015;12:592-605.

86. Duff EP, Vennart W, Wise RG, et al. Learning to identify CNS drug action and efficacy using multistudy fMRI data. Sci Transl Med 2015;7:274ra16

87. McGrath CL, Kelley ME, Holtzheimer PE, et al. Toward a neuroimaging treatment selection biomarker for major depressive disorder. JAMA Psychiatry 2013;70:821-829.

88. Wong RK, Van Oudenhove L, Li X, Cao Y, Ho KY, Wilder-Smith CH. Visceral pain perception in patients with irritable bowel syndrome and healthy volunteers is affected by the MRI scanner environment. United European Gastroenterol J 2016;4:132-141.

89. Sundermann B, Herr D, Schwindt W, Pfleiderer B. Multivariate classification of blood oxygen level-dependent fMRI data with diagnostic intention: a clinical perspective. AJNR Am J Neuroradiol 2014;35:848855. 